\title{
Constructing the Long-Distance Speech Rehabilitation Service Process for Patients with Parkinson's Diseases
}

\author{
Hsin-Chang Lo ${ }^{1+}$, Ching-Min Chang ${ }^{2}$, Shih-Tsang Tang ${ }^{3}$ and Tsorng-Lin Chia ${ }^{4}$ \\ ${ }^{1,2}$ Department of Product Design; ${ }^{3}$ Department of Biomedical Engineering; \\ ${ }^{4}$ Department of Computer and Communication Engineering, Ming Chuan University, Taiwan
}

\begin{abstract}
Around 90\% of patients with Parkinson's disease (PD) experience speech disorders. Most patients with PD do not continue practice at home but only go to the rehabilitation center for treatments, therefore the rehabilitation effect is poor. This study aimed to construct, with the service design method, a long-distance speech rehabilitation service process for them. Three patients were recruited to participate in the empirical study to operate two types of mobile application software (APP) related to speech therapy and three types of voice recording APP. The researcher observed and interview with subjects to explore the problems that subjects might encounter when using the APPs in tablet PC. From the site-observation and indepth interview with patients with PD it found some defects when operating the above-list APPs. Then this study developed the service blueprint and service process of the long-distance speech rehabilitation base on the empirical study results. The research team will develop a speech rehabilitation APP and platform using the information and communication technology in the next stage.
\end{abstract}

Keywords: service design, speech rehabilitation, parkinson's disease.

\section{Introduction}

Progressive neurological diseases, such as Parkinson's disease (PD) impair the motor functions, such as speech, swallowing, limb function, gait, balance, and activities of daily living. Even with pharmacological, surgical treatments these deficits cannot be controlled satisfactorily in the vast majority of patients with PD and have a negative impact on quality of life [1]. About $90 \%$ of patients with PD suffer from speech disorder, especially in dysarthria, because of their muscle control ability declines [2]. Their voice commonly features monopitch, feebleness, slowness, incoordination, hoarseness and irregular articulatory breakdown. The speech disorder gets worse with time but can be significantly improved by speech rehabilitation. Additionally, about 50\% of patients with PD suffered from swallow problems. During a meal, food or liquid overflows from their mouth or food cannot be swallowed and get stuck in the throat, which causes coughing, choking regurgitation and aspiration. Swallow problems make it difficult for the patients to clean their lung by expectoration and thus increase the risk of getting pneumonia [2]. Pronunciation and swallow normally depend on the muscle groups in the pharynx and larynx, these can be improved effectively by speech rehabilitation. Traditionally, speech rehabilitation refers to a series of face-to-face treatments by the speech and language therapist, including pronunciation exercises, speaking speed control, articulation exercises, emotional expression, tone exercises, volume control and breath control. Such auxiliary equipment as the sound feedback device, the amplifier and the rhythm device will be used as necessary [3, 4]. Most patients with PD do not continue practice at home but only go to the rehabilitation center for treatments, therefore the rehabilitation effect is poor. Caregivers and therapists must constantly persuade, urge and guide the patients to go on with rehabilitation in other places apart from rehabilitation center [5]. Regarding the difficulties mentioned above, integrating the information and communication technology into the long-distance care

\footnotetext{
${ }^{+}$Corresponding author. Tel.: +886-3-3507001 ext 5036; fax: +886-3-3593882.
}

E-mail address: lohc@mail.mcu.edu.tw. 
service may be able to bring feasible solutions to patients with PD [6]. Therefore, this study aimed to construct, with the service design, a long-distance speech rehabilitation service process for patients with PD.

\section{Empirical Study}

Three patients with PD with Hoehn-Yahr stages I to III were recruited to participate in the empirical study (Fig. 1). The detailed information was shown in Table 1. Two types of mobile application software (App) related to speech therapy (Fig. 2) and three types of voice recording APP were downloaded from Google Play (formerly Android Market) to Sony Xperia ${ }^{\mathrm{TM}}$ Z4 Tablet PC (Android 5.0, 10.1", WQXGA $2560 \times 1600$ pixel). The researcher observed the subjects' operational behaviors of the five above-listed Apps and inquired them about their reflections on operation after the test to explore the defects the subjects might encounter when using the tablet for speech therapy.
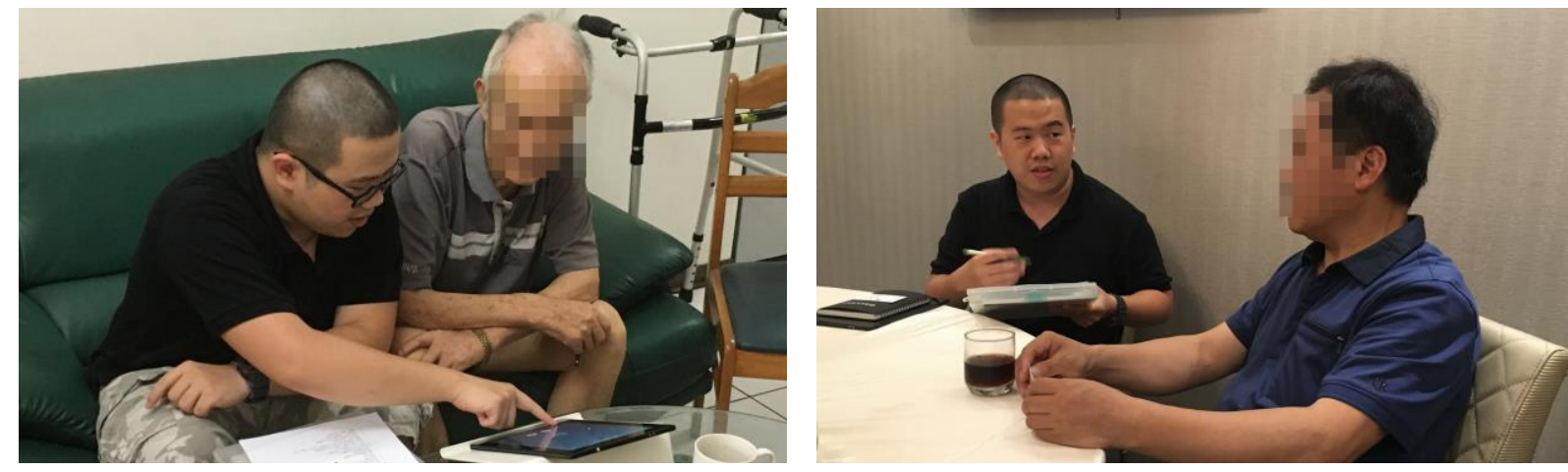

Fig. 1: Subject No.2 and No.3 participated in this study.

Table 1: Description of the subjects

\begin{tabular}{lllllll}
\hline No & Sex & Age & Education & Visual ability & Tablet Experience & Hoehn-Yahr Stages \\
\hline 1 & Male & 78 & Less than high school & $\begin{array}{l}\text { Presbyopia, } \\
\text { cataract }\end{array}$ & No & I \\
2 & Male & 84 & Bachelor & $\begin{array}{l}\text { Presbyopia, } \\
\text { cataract }\end{array}$ & No & III \\
presbyopia & Yes & II \\
\hline
\end{tabular}

${ }^{a}$ received deep brain stimulation

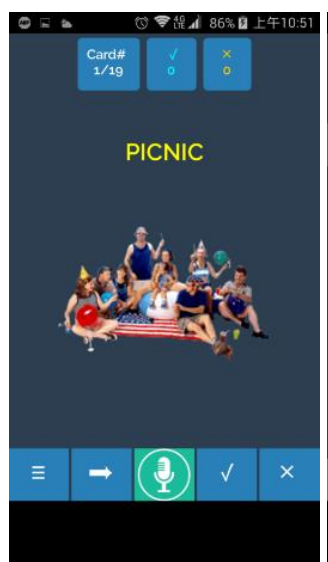

(a)

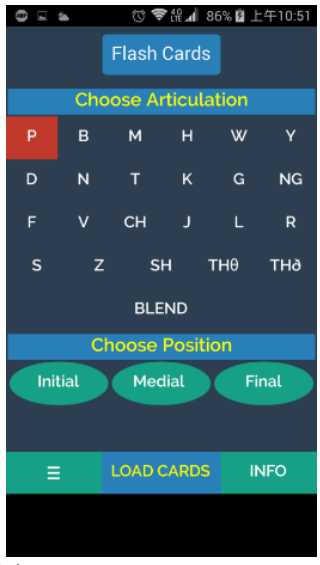

(a)

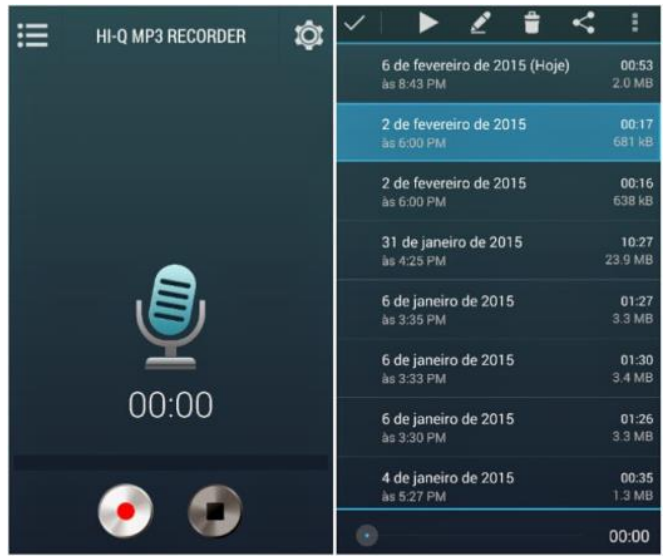

(b)

Fig. 2: Selected Apps in the empirical study for example: (a) Speech Therapy App; (b) Voice recorded App.

\section{Results}

\subsection{The Results of Empirical Study}


Through empirical study, we found that the patients with PD were difficult to understand the meaning of current speech therapy and voice recording Apps image symbol (icon), such as play, forward, record, etc. Most of them represent that they need Chinese description, otherwise they will speculate the meaning of icon to operate the task. Because of the education level, we believed that the text instruction, especially in Chinese, should be needed in the operation process to avoid forgetting the steps that have been performed or to be execution. In addition, the size of the icon and the text must be increased, the hue or brightness contrast must also be strengthened, therefore can facilitate user reading identification, and icon touching. For the subjects who use of the touch interface in the first time, the accurate click operation is not so easy. Therefore, the appearance of icons and text is best not to have much change after clicking the icons. The subjects were not familiar with the operation of the tablet PC, they will take the "from where to start from where to end" strategy to operate it. If the icon or text changes too much, it may lead to confusion easily. Finally, the file naming problem. Most of the recorded files were named after the date and time of the recording, such as "2015.11.21 11:31 am", it is quite inconvenient for the subjects to find the relevant recording material. If the subjects did not remember the file recording time, it is difficult to find the correct recording files, especially for those who can not understand the naming logic. They were completely do not understand, or even simply do not know that the specific file is the one that they just recorded.

\subsection{Building the Service Blueprint}

This study summarized the empirical study results and build the speech rehabilitation service blueprint for patients with PD (Fig. 3).

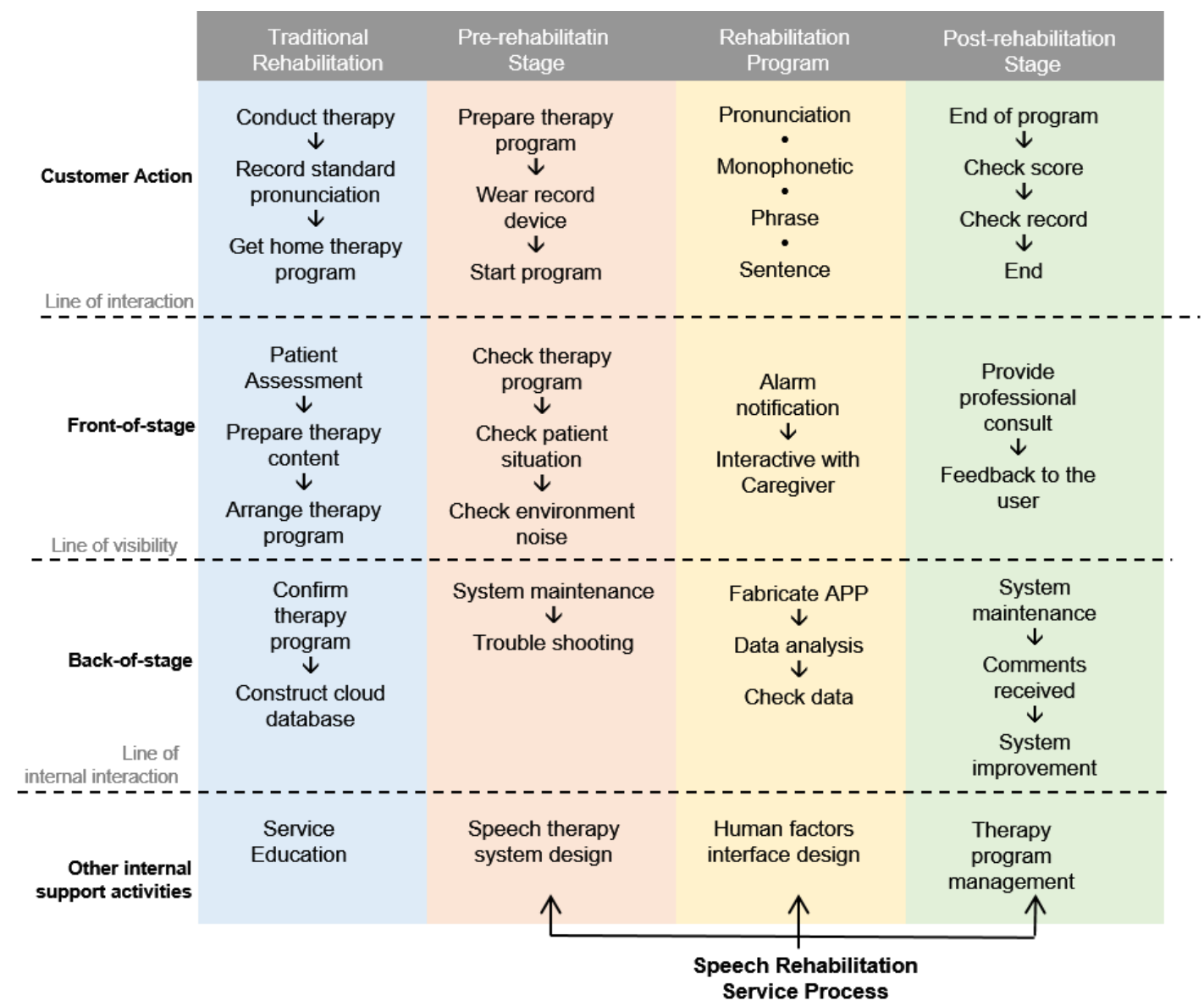

Fig. 3: The blueprint of speech rehabilitation for the patients with PD

Through the lines of interaction, visibility, internal interaction, the whole service process was divided into four sections: customer action, front-of-stage, back-of-stage, and other internal support activities [7]. This blueprint focused on the service received by the patients and the performance of the front-of-stage interaction. So the speech rehabilitation service actions were classified into the traditional rehabilitation, pre- 
rehabilitation stage, rehabilitation program, and post- rehabilitation stage. This study did not investigate the interaction of the back-stage and other supporting units.

\subsection{Constructing the Service Structure of Speech rehabilitation}

This study then constructed the speech rehabilitation service structure for patients with PD (Fig 4). There are six categories includes Main, Program, Rehabilitation effects, Pay service fee, Database, and Other service. The most important is Program category. When the service receiver (patients with PD) started the APP on the tablet PC, it would first test the background noise of the practice environment. The speech and language therapist suggested $50 \mathrm{~dB}$ as the critical value [8]. If the background noise exceeded $50 \mathrm{~dB}$, users would be advised to do the practice in other field. If the background noise was acceptable, the user would enter the main menu and select the target item from: a. Pronunciation, $b$. Monophonetic (including the volume, persistence, high pitch and low pitch), c. Phrase, d. Sentence (including the volume and intelligibility). The users could exercise the same item repeatly. At the end, it would provide the service receiver with the practice results in the of Rehabilitation effects category. This service was expected to provide: $a$. the volume score, $\mathrm{b}$. the intelligibility score, and $\mathrm{c}$. the comprehensive score as a reference for the user. The comprehensive score was weighted from the volume and intelligibility scores. Meanwhile, the practice results would be compared with the personal reference obtained in traditional speech rehabilitation to show whether any progress was made in the current practice. At last, the rehabilitation results would be recorded.

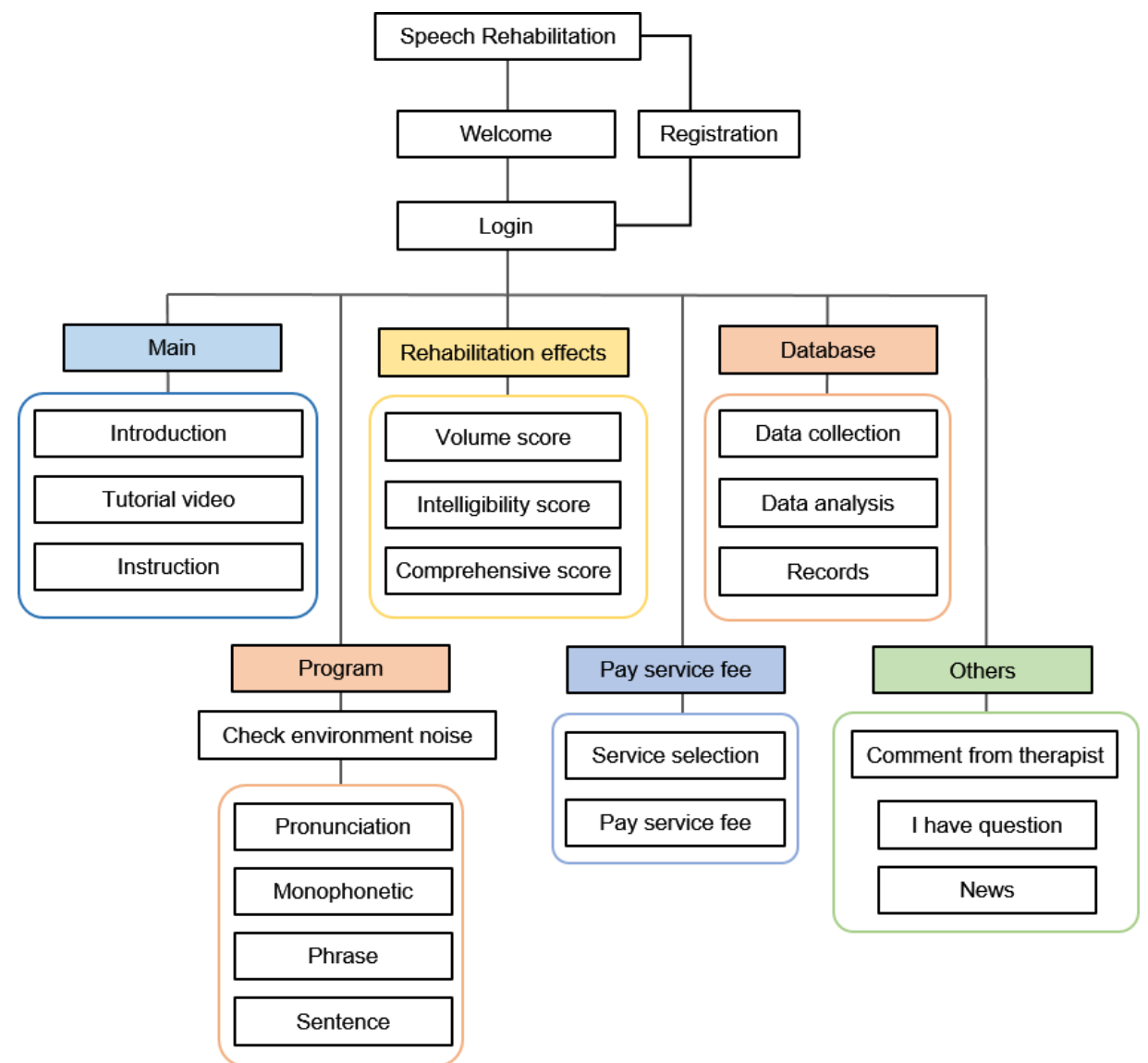

Fig. 4: The service process of Speech Rehabilitation for the patients with PD

\section{Discussion and Conclusion}

Due to poor balance, the patients with PD are not willing to go out for the traditional face-to-face speech rehabilitation in the rehabilitation center, which may reduce their rehabilitation effect. From the service design perspective, this study emphasized the method to cooperate with service receivers. The patients with PD were invited to operate, via the tablet PC, the speech therapy and the recording Apps. The siteobservation and in-depth interview were adopted to develop the service blueprint and service process of the long-distance speech rehabilitation for patients with PD. In the next stage, the research team will develop a 
speech rehabilitation platform on the tablet PC with the information and communication technology and the cloud computation technology. The constructed long-distance speech rehabilitation service can not only serve as an auxiliary tool for the speech and language therapist, but also improve the effect of home rehabilitation. The therapist is thus able to grasp the quality and quantity of the patients' off center rehabilitation at any time. Therefore, it will be of important significance to improve the patients' life quality.

\section{Acknowledgements}

The authors appreciate the participation of patients with PD. This work was sponsored under grant MOST 104-2410-H-130-048 - and 104-2632-E-130-001 - by the Ministry of Science and Technology, Taiwan.

\section{References}

[1] P. A. Crawford. The Washington manual cardiology subspecialty consult, Lippincott Williams \& Wilkins, Philadelphia, 2003.

[2] F. Cynthia, E. Georg, R. Lorraine, S. Shimon. LSVT LOUD and LSVT BIG: Behavioral Treatment Programs for Speech and Body Movement in Parkinson Disease, Parkinsons Dis. 2012, 391946.

[3] W. J. Weiner, C. Singer. Parkinson's disease and nonpharmacologic treatment programs. J. Am. Geriatr. Soc. 1989, 37: 359-363.

[4] E. Aronson. Clinical Voice Disorders. Thieme, New York, 1990.

[5] C. Ellis-Hill, J. Robison, R. Wiles, K McPherson, D. Hyndman, A. Ashburn. Going home to get on with life: patients and careers experiences of being discharged from hospital following a stroke, Disability and Rehabilitation, 31(2009) 61-72.

[6] F. Pasluosta, H. Gassner, J. Winkler, J. Klucken, B. M. Eskofier. An Emerging Era in the Management of Parkinson's Disease: Wearable Technologies and the Internet of Things, IEEE J. Biomed. Health Inform. 2015, 19: 1873-1881.

[7] V. A. Zeithaml, M. J. Bitner, D. D. Gremler, Services marketing: integrating customer focus across the firm. McGraw-Hill/Irwin, Boston, 2006.

[8] J. Baken, and R. F. Orlikoff, Clinical measurement of speech and voice, 2nd ed. Singular Thomson Learning, San Diego, 2000. 